\title{
A Novel Study on Design to Reduce PAPR and ISI in Multi-Carrier OFDM System
}

\author{
Jyoti Makkar \\ M.Tech Scholar \\ Electronics \& Communication Deptt. \\ Jan Nayak Ch. Devi Lal Vidyapeeth \\ Sirsa-125055, Haryana, India
}

\author{
Himanshu Monga, PhD \\ Professor \\ Electronics \& Communication Deptt. \\ Jan Nayak Ch. Devi Lal Vidyapeeth \\ Sirsa-125055, Haryana, India
}

\begin{abstract}
This paper provides a review on a new scheme for PAPR reduction in OFDM System. OFDM system mainly used to provide orthogonality of OFDM signals. This system used Fast Fourier Transform (FFT) for modulation and inverse Fast Fourier Transform (IFFT) for demodulation purposes. In this paper, we will discuss the overview of OFDM system which consist OFDM system model and notation, OFDM transmitter and its receiver structure, concepts of orthogonality. It also describes inter-symbol interference problem, high peak-toaverage power ratio (PAPR) problem and generate a taxonomy of the available solutions to mitigate the problem. The high PAPR will cause the degradation of performance of system and hence high order filter will be implemented in system using MATLAB tool.
\end{abstract}

\section{Keywords}

OFDM System, PAPR Reduction, ISI method etc.

\section{INTRODUCTION}

The everyday dynamic revelation of expanded mixed media remote applications and gadgets has prompted a solid interest for innovation that can give fast information transmission and furthermore use the accessible assets productively. The innovation that can satisfy every one of the requests for high information transmission and furthermore use the accessible transfer speed assets adequately is the Orthogonal Frequency Division Multiplexing (OFDM) innovation [1]. Look into in light of OFDM innovation was first distributed by Robert W. Chang in the year 1966 [2].

The sub-transporters in the OFDM flag are orchestrated such that the sidebands of the individual sub-bearer cover with each other (Fig. 1) and the relating yield flag can be gotten with no adjoining transporter impedance. Hence, there is an effective usage of the data transfer capacity in the OFDM system [3]. Fundamentally OFDM is a multicarrier transmission framework wherein a solitary higher rate information transporter is separated into a substantial number of parallel lower rate information bearers which are exclusively called as sub-transporters, subsequently lessening the Inter image Interference (ISI) because of huge image length not at all like the customary Frequency Division Multiplexing (FDM) innovation [4]. Because of every one of these benefits, OFDM has been embraced as the standard decision for future fast correspondence. Additionally consolidated in the IEEE 802.11a and IEEE $802.11 \mathrm{~g}$ for rapid information transmission.

Different applications and guidelines in which OFDM has been consolidated is the Digital Audio Broadcasting (DAB), Digital Video Broadcasting (DVB) and so forth [5], [6]. In a remote communicate framework, OFDM assumes a critical part and decrease the multifaceted nature of beneficiary however in this procedure channel estimation and synchronization is vital [7]. The OFDM framework is power to blurring which is created because of multi-way obstruction. $\mathrm{CP}$ is added to every image to conquer the ISI impact which prompts high phantom effectiveness however it likewise cause swells in power unearthly thickness [8]. Nonetheless, one of the real downsides of the OFDM is the high PAPR which emerges due to the IFFT operation. This high Peak to Average Power Ratio (PAPR) esteem makes the intensifier qualities move in nonlinear district of operation, because of which OFDM framework loses its orthogonality prompting extreme Inter transporter obstruction (ICI) [9]. In this writing audit paper, a few calculations for diminishing the PAPR esteem have been illustrated. One of the upsides of OFDM is that it accomplished an adequate level channel because of an expansive number of subcarriers and it likewise accomplished a same information rate of single transporter tweak at a most minimal image rate. The images turn out to be longer because of the drop of image rate of every bearer. The fig 1 describes the spectral efficiency of FDM and OFDM system.

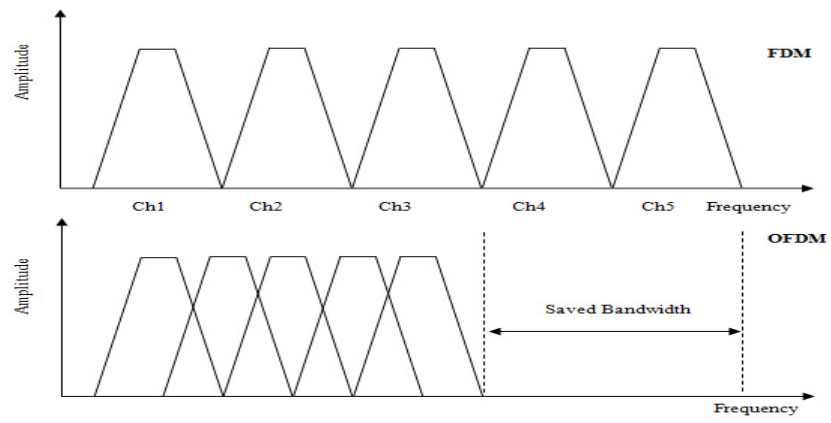

Fig 1: Spectral Utilization Efficiency Comparison between FDM and OFDM Schemes

The paper is planned as follows. In section II, it provides the related work with the design and implementation of OFDM transceiver, ISI and PAPR problem and its reduction technique. In Section III, It describes OFDM system model and its notations, which also consists OFDM transmitter and its receiver design. In Section IV, it describes the introduction of ISI and PAPR problem and its reduction techniques. Finally Section V describes the conclusion of system.

\section{RELATED WORK}

In 2016, Saravana Rathinakumar et.al [10] proposed the work on "CPRecycle: Recycling Cyclic Prefix for Versatile Interference Mitigation in OFDM based Wireless Systems". In this work, it proposed novel OFDM PHY beneficiary outline, called CPRecycle, that exploited the repetitive bit of the $\mathrm{CP}$ towards impedance relief. In particular, CPR cycle models the impact of impedance in each subcarrier utilizing a Gaussian bit 
thickness work utilizing the introduction images and used a settled circle greatest probability identifier to decipher the accompanying information conveying OFDM images subject to obstruction. Utilizing off-the-rack IEEE $802.11 \mathrm{~g}$ transmitters and interferers, we tentatively demonstrate the adequacy of CPRecycle for relieving neighbouring channel impedance and co-channel obstruction. We likewise demonstrate that two preludes and little part of $\mathrm{CP}$ are adequate to acknowledge critical advantages as far as parcel achievement rate with CPRecycle. The use of CPRecycle in numerous reception apparatus frameworks and assessment of CPRecycle within the sight of various sorts of obstruction is left for future work.

In 2016, Rakeshkumar Saroj [11] proposed "A Cooperative Additional Hybrid and Clipping Technique for PAPR decreases in OFDM System". In this work, it had proposed different calculations, for example, Additional Hybrid method, Alternate Additional Hybrid procedure and Cooperative Additional Hybrid strategy for finding the PAPR esteem in the OFDM framework. After figuring of the PAPR values for the Alternate and Cooperative Additional Hybrid system, it had connected the Clipping procedure after IFFT in the Cooperative Additional Hybrid strategy to smother the abundance tops from the OFDM flag waveform subsequently additionally lessening the PAPR esteem.

In 2016, Panca Dewi Pamungkasari et.al [12] proposed "Move Estimation with Delayed Correlation Cyclic-Selective Mapping (Cyclic-SLM) PAPR Reduction in OFDM System". In this work, the deferred relationship based estimation plan was proposed to evade the transmission of the side data (SI). This plan misused the relationship between's a monitor interim succession. Numerical outcomes gotten through PC reproduction demonstrate that the proposed plan can gauge the deferral with an exactness of more than 0.99 at an $\mathrm{Eb} / \mathrm{No}$ of $8 \mathrm{db}$ and accomplish the PAPR that is proportionate to the customary SLM while the BER corruption is somewhat unique in relation to that with immaculate move estimation.

In 2016, Mamadou Lamarana Diallo et.al [13] proposed the work on "Changed Tone Reservation for PAPR Reduction in OFDM Systems". The proposed technique accomplishes a change up to $1.2 \mathrm{~dB}$ as far as PAPR execution without expanding the many-sided quality. The viability of this arrangement is affirmed through hypothetical examination and recreation comes about. Besides, it had demonstrated that going profoundly in the deterioration procedure of the helpful OFDM flag accomplishes better execution for the MTR-GP strategy.

In 2016, Md Sakir Hossain et.al [14] proposed a "Low complexity invalid sub-caarier-helped OFDM PAPR lessening with enhanced BER". Because of the high intricacy and poor BER of the traditional information invalid subcarrier exchanging based PAPR lessening plan, proposed an enhanced PAPR diminishment conspire, with low computational overhead, which can reduction such impacts drastically. So the proposed plot lessens the framework intricacy by over $98 \%$ and accomplishes up to $6 \mathrm{db}$ SNR pick up.

In 2015, Anusha Chacko et.al [15] proposed the work on "Proficient Use of PTS for PAPR Reduction in OFDM System". In this work, it had proposed two strategies which productively utilize PTS, PTS with division and PTS with interleaving. High PAPR is the major testing issue in OFDM frameworks. Halfway Transmit Sequence procedure is a productive strategy to farthest point high PAPR. The strategies brought about further lessening in PAPR than the ordinary PTS.
In 2014, K. Sangeetha et.al [16] proposed the work on "Plan and Implementation of OFDM Transceiver for ISI Reduction utilizing Sinc Filter". In this work, built up an OFDM handsets in light of the proposed design which accomplishes over $2 \mathrm{Gbps}$ The outcomes demonstrate that the outline has been reproduced up to $227.355 \mathrm{MHz}$ and it accomplishes higher speed and lower region by utilizing Radix-2 parallelized 1024 point FFT/IFFT calculation utilizing Mat lab. It is utilized to diminish the ISI Value utilizing Sinc Filter. This expands its speed and zone element is additionally dealt with. The proposed configuration has indicated change in territory consider additionally terms of no. of cuts and no. of multipliers. Also the proposed configuration has been executed on multipliers based lower end FPGAs keeping in mind the end goal to give financially savvy answer for remote correspondence applications.

In 2013, Abhijit et.al [17] proposed the work on "OFDM framework utilizing FFT and IFFT" The proposed work will be to plan the 32-point FFT and IFFT obstructs for OFDM by utilizing VEDIC augmentation and to accomplish the productive duplication. The outline would be coded in VHDL and union was done in Xilinx ISE programming. At that point the plan will get actualized on FPGA pack. In this manner, we can get the proficient duplication of FFT and IFFT square to be utilized as a part of OFDM recipient and transmitter individually.

\section{OVERVIEW OF OFDM SYSTEM}

\subsection{OFDM System Model}

An OFDM can be created utilizing various tweaked transporters transmitted in parallel. Be that as it may, this technique includes usage issues and makes trans-receiver more perplexing and costly. This issue can be evaded by the utilization of discrete Fourier change strategy The OFDM balanced flag is given by taking after scientific expression [18]:

Consider a data stream having $\mathrm{R}$ rate bps where data bits are mapped to several group of stars focuses utilizing an advanced adjustment similar to the quadrature abundancy regulation (QAM). Let $\mathrm{N}$ of these star grouping focuses be put away for an interim of $T \_s=N / R$, alluded to as the OFDM image interim. A serial-to-parallel converter is utilized to accomplish this. Presently, every one of the $\mathrm{N}$ group of stars focuses is utilized to regulate one of the subcarriers, then, all balanced subcarriers are transmitted all the while over the image interim T_s [19]. The OFDM flag $\mathrm{x}(\mathrm{t})$ can be communicated is given by eq. (1):

$$
\begin{aligned}
& x(t)=\sum_{k=0}^{N-1} a_{k} \exp \left(j 2 \pi\left(f_{c}+k \Delta f\right) t\right) \\
& =\exp \left(j 2 \pi f_{c} t\right) \sum_{k=0}^{N-1 a^{k}} \exp (j 2 \pi k \Delta f t) \\
& =\exp \left(j 2 \pi f_{c} t\right) a(t),
\end{aligned}
$$

where $a k, 0 \leq k \leq N-1$ are complex-valued constellation data and $f k=f c+k \Delta f, 0 \leq k \leq N-1$, is the kth subcarrier and $f c$ being the lowest subcarrier frequency. $\Delta f$ is the frequency spacing between adjacent subcarriers, chosen to be $1 /$ Ts to ensure that the subcarriers are orthogonal. If $a(t)$ is sampled at rate $R$ samples per second, where $t$ is replaced by $\frac{n T s}{N}, n=0, \ldots N-1$, then $a(t)$ is represented by the sampled function $a[n]$ expressed as: 


$$
a[n]=\sum_{k=0}^{N-1} a_{k} \exp \left(\frac{j 2 \pi k n}{N}\right)
$$

This condition takes the very same shape as the opposite discrete Fourier change (IDFT) and can be actualized effectively utilizing the backwards quick Fourier change (IFFT) calculation [20]. Conditions (1) and (2) exhibit that OFDM can be produced by regulating the IFFT of the grouping, $\{\mathrm{a}[\mathrm{n}], 0 \leqslant \mathrm{n} \leqslant \mathrm{N}-1\}$ by a solitary transporter of recurrence $\mathrm{fc}$ rather than by adjusting $\mathrm{N}$ heavenly body focuses by subcarriers. IFFT decreases the computational intricacy in contrast with IDFT. DSP chip executions of IFFT and FFT are promptly accessible. The above perception drives us to presume that IFFT ought to be the favored decision for OFDM frameworks usage. Subsequent to demodulating the got flag, the recipient completes the turnaround procedure of that of the transmitter amid each OFDM image interim by utilizing FFT, a parallel-to-serial converter, and a demapping to recuperate the coveted information bit stream [3].

\subsubsection{OFDM Transmitter}

The approaching serial information is the data that should be transmitted through the channel utilizing OFDM framework. The serial in changed over into $\mathrm{N}$ distinctive parallel information streams by utilizing serial to parallel converter. These images can be adjusted by utilizing distinctive balance methods and given to the IFFT hinder as an information. IFFT piece gives the computerized time space motion for the given info, and this parallel information is changed over into serial information by utilizing parallel to serial converter. The cyclic prefix is acquainted between two OFDM images with scratch off the impact of ISI because of channel scattering. Presently this computerized time flag is changed over into ongoing waveform with the utilization of advanced to simple converters. The accessible baseband flag is up changed over to RF passband motion with the utilization of blender or modulators [19].

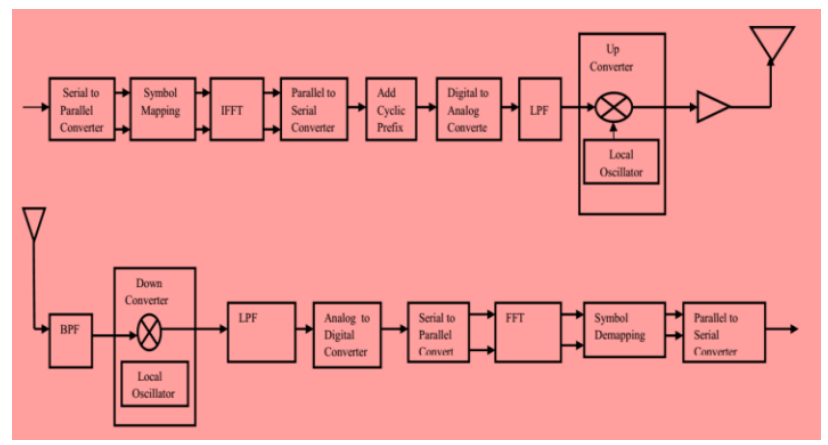

Fig 2: Conventional FFT-based OFDM transmitter and receiver

\subsubsection{OFDM Receiver}

At the beneficiary end, the got OFDM flag is down changed over utilizing the demodulator and examined with simple toadvanced converters to acquire the computerized time space flag. The computerized time space flag is demodulated by utilizing FFT, and the information that is transmitted can be separated by utilizing image demapper [19]. The OFDM transmitter and beneficiary is appeared in beneath Figure 2. It demonstrates a traditional FFT-based OFDM framework, where IFFT and FFT are utilized at the transmitter and recipient separately.

\subsubsection{Concept of orthogonality}

In OFDM, the sub-transporter frequencies are picked so that the sub-bearers are orthogonal to each other. It implies that cross-talk between the sub-channels is wiped out and between transporter monitor groups are not required. This extraordinarily rearranges the plan of both the transmitter and the collector; not at all like regular FDM, is a different channel for each sub-channel not required. The orthogonality [21] requires that the sub-carrier spacing is $\Delta f=\frac{K}{T U}$ Hertz, where TU seconds is the useful symbol duration, and $\mathrm{k}$ is a positive integer, usually equal to 1 . Therefore, with $\mathrm{N}$ sub-carriers, the total passband bandwidth will be $=\approx \mathrm{N} \Delta \mathrm{f}(\mathrm{HZ})$.

\section{PROBLEM FORMULATION}

\subsection{Peak to average power ratio (PAPR)}

A noteworthy burden that emerges in multicarrier frameworks like OFDM is the subsequent non-consistent envelope with high pinnacles [22]. At the point when the autonomously adjusted subcarriers are included soundly, the quick power will be more than the normal power.

\subsubsection{Definition of PAPR}

The PAPR for the continuous-time signal $x(t)$ is the ratio of the maximum instantaneous power to the average power. For the discrete-time version $x[n]$, PAPR is expressed as:

$$
\operatorname{PAPR}(x[n])=\left(\frac{\max \left\{|x[n]|^{2}\right\}}{E\left\{|x[n]|^{2}\right\}}\right),
$$

where $E[$.$] is the expectation operator. It is worth mentioning$ here that PAPR is evaluated per OFDM symbol.

$x(n)$ represent the amplitude of the complex pass band signal. Such high peaks will produce signal excursions into nonlinear region of operation of the power amplifier (PA) at the transmitter, thereby leading to nonlinear distortions and spectral spreading.

\subsection{Inter-symbol interference (ISI) problem}

Among the distinctive sorts of bends in correspondence Inter Symbol Interference is a wonder that causes the substantial information misfortune in the correspondence. Typically the advanced data that is transmitted will be as square waveform speaking to the 1 's and 0 's. At the point when this square waveform blends with the commotions and non linearity's in the channel, the square waveform begins to spread and converge with the neighboring image succession, making the information there to be indiscernible [23].

At the collector end this information is wrongly decoded as the beneficiary can't foresee the right level of the square waveform prompting the loss of data. The Fig.3 speaks to the graphical portrayal of ISI. The nearness of ISI in the framework presents mistakes in the choice gadget at the collector yield. Along these lines, in the outline of the transmitting and getting channels, the goal is to limit the impacts of ISI, and consequently convey the advanced information to its goal with the littlest blunder rate conceivable.

\subsubsection{Causes of inter symbol interference}

- Multipath propagation

- Band-limited channels

- Noises and Fading Channels 


\subsubsection{Protection against ISI}

There are a few strategies and information stockpiling that attempt to work around the issue of between image obstructions. Plan frameworks to such an extent that the drive reaction is sufficiently short that almost no vitality from one image smears into the following image. Isolate images in time with protect periods. Apply an equalizer at the recipient that extensively, endeavors to fix the impact of the channel by applying a reverse channel.

Apply a grouping indicator at the recipient that endeavors to gauge the arrangement of transmitted images utilizing the Viterbi calculation. Plan frameworks with the end goal that the drive reaction is sufficiently short that almost no vitality from one image smears into the following image.

ISI impact can be lessened by intriguing a watch interim (cyclic prefix). The cyclic prefix or protect interim is an intermittent expansion of the last area of an OFDM image that is added to the forepart of the image in the transmitter, and is expelled at the beneficiary before demodulation.

The cyclic prefix has two critical advantages

1. The cyclic prefix goes about as a protect interim. It dispenses with the between image impedance from the past image [14].

2. It goes about as a redundancy of the finish of the image along these lines permitting the liner convolution of a recurrence particular multipath channel to be demonstrated as roundabout convolution which thus might be changed to the recurrence area utilizing a discrete Fourier Transform. This approach takes into consideration straightforward recurrence area preparing, for example, channel estimation and balance.

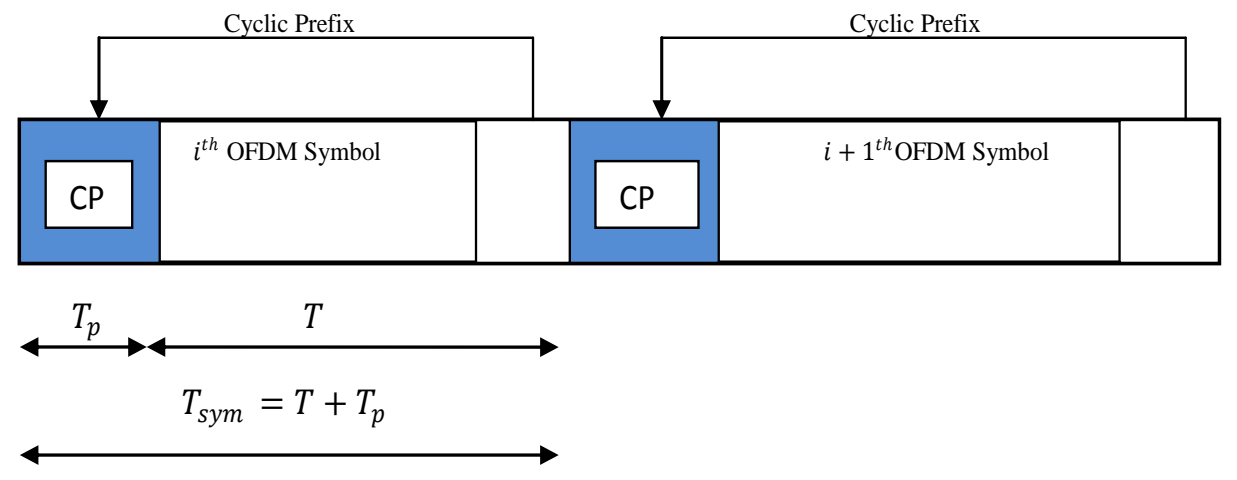

Fig 3: Cyclic Prefix

\section{CONCLUSION}

In this paper, it presents a review on OFDM system for reducing PAPR value. OFDM has newly found wide assumption in a wide variety of high data communication system. In this work, it will provide the information about reduction of PAPR value in system. For this, system will be realized based on FFT and IFFT transform methods. PAPR basically occur when in the multicarrier system the different sub-carrier is out of phase from each other. It works on filtering technique in OFDM system for controlling PAPR value. Adding guard interval in signal transmission with orthogonal subcarriers, interference inter-symbols in channel can be eliminated and hence ensured safe and effective transmission of information in communication process. All simulations will be implemented in MATLAB.

In future, spatial coding will be used to design OFDM system for reducing further PAPR value and suitable fuzzy logic will be used to enhance the system performance.

\section{REFERENCES}

[1] Yasir Rahmatallah and Seshadri Mohan, "Peak-ToAverage Power Ratio Reduction in OFDM Systems: A Survey And Taxonomy," IEEE Commun. Surveys and Tutorials, vol. 15, issue 4,pp. 1567-1592,March 2013.

[2] LaSorte, N, Barnes, W. J, Refai, H. H. , "The History of Orthogonal Frequency Division Multiplexing," in Global Telecommunications Conference, 2008. IEEE GLOBECOM 2008. IEEE, pp.I-5, December, 2008.

[3] Louis, L. and P. Michael, 2001. Signal processing.
[4] Ramjee Prasad, -OFDM for wireless communication system", Artech House, 2004.

[5] R. V. Nee, G. Awater, M. Morikura, H. Takanashi, M. Webster, and K. W. Halford, "New high-rate wireless LAN standards," IEEE Commun. Mag., vol. 37, no. 12, pp. 82-88, December1966.

[6] I. Koffman and V. Roman, "Broadband wireless access solution based on OFDM access in IEEE 802.16," IEEE Commun. Mag., vol. 40, no. 4, pp. 96-103, April 2002.

[7] Vani, M.D. and B.S. Reddy, 2013. Design and BER performance of MIMO-OFDM for wireless broadband communications. Int. J. Modern Eng. Res., 3: 1382-1385.

[8] Urmila, S. and R.C. Patel, 2014. Design and implementation of OFDM transmitter and receiver using 8-point FFT/IFFT. Int. J. Social Relevance Concern, 2: 236.243 .

[9] Tao, J. and Y. Wu, 2008. An overview: peak-to-average power ratio reduction techniques for OFDM signals. IEEE Trans. Broadcast., 54: 257-268. DOI: 10.1109/TBC.2008.915770.

[10] Saravana Rathinakumar, Bozidar Radunovicy, Mahesh K. Marina, "CPRecycle: Recycling Cyclic Prefix for Versatile Interference Mitigation in OFDM based Wireless Systems" In Proceedings of the ACM MobiCom (2016), DOI: 10.1145/2999572.2999577.

[11] Rakeshkumar Saroj, "Cooperative Additional Hybrid and Clipping Technique for PAPR reduction in OFDM 
System" IEEE Fifth International Conference on EcoFriendly Computing and Communication Systems (ICECCS), 2016.

[12] Panca dewi pamungkasari, yukitoshi sanada, "shift estimation with delayed correlation cyclic-selective mapping (cyclic-SLM) PAPR reduction in OFDM system" wireless pers commun, springer 2016.

[13] Mamadou Lamarana Diallo, Marwa Chafii, Jacques Palicot and Faouzi Bader, "Modified Tone Reservation for PAPR Reduction in OFDM Systems" 24th European Signal Processing Conference (EUSIPCO) 2016.

[14] Md sakir hossain, tetsuya shimamura, ““'Low -complexity null subcaarier-assisted OFDM PAPR reduction with improved BER" inI IEEE comm.. letters, DOI:10.1109.2016.2598801, 2016.

[15] Anusha Chacko, Grace John M, J. Jeevitha, "Efficient Use of PTS for PAPR Reduction in OFDM System" in International Journal of Engineering Trends and Technology (IJETT) Volume 22, 2015.

[16] k.sangeetha, m.venkatesan, "Design and Implementation of OFDM Transceiver for ISI Reduction using Sinc Filter" in International Journal o f Emerging Technology in Computer Science \& Electronics (IJETCSE), Volume 8 Issue 12014.

[17] Abihijit D. Palekar, Dr. Prashant V. Ingole, “ Ofdm System Using FFT and IFFT" in International Journal of Advanced Research in Computer Science and Software Engineering,Volume 3, 2013.
[18] H. Schulze and C. luders, Theory and Applications of OFDM and CDMA. John Wiley \& Sons Ltd., 2005.

[19] M. Schwartz, Mobile Wireless Communications. Cambridge University Press, 2005.

[20] A. V. Oppenheim and A. S. Willsky, Signals and Systems. Upper Saddle River, NJ, Prentice-Hall, 1997.

[21] G.Banupriya, D.Thilagavathi, "Features and Principles of OFDM: A Brief Study", International Journal of Innovative Research in Computer and Communication Engineering, Vol. 4, Issue 8, August 2016.

[22] V. Tarokh and H. Jafarkhani, "On the computation and reduction of the peak-to-average power ratio in multicarrier communications," IEEE Trans. Commun., vol. 48, pp. 37-44, 2000.

[23] Lathi, B.P.; Ding, Zhi (2009). Modern Digital and Analog Communication Systems (Fourth ed.). Oxford University.

\section{AUTHOR PROFILE}

Jyoti makkar received the B. Eng. degree from Eternal University, Akal college of baru sahib in 2014 and pursuing M. Eng. degree from Guru jamdeshwar University, JCDMCOE, both in electronics and communication engineering. I am currently working in the area of signal processing for wireless communication and research interests include OFDM communication systems and wireless networking. 